O filme codificado ${ }^{1}$

Gabriel Menotti²

1 Esse artigo é uma versão traduzida e ampliada de um trecho do livro Movie Circuits: Curatorial Approaches to Cinema Technology, do autor, e foi publicado com a autorização da Amsterdam University Press.

2 Professor do Departamento de Comunicação da Universidade Federal do Espírito Santo e dos Programas de Pós-Graduação em Artes e Comunicação e Territorialidades da mesma instituição. Coordena a rede de pesquisa Besides the Screen.

e-mail: gabriel.menotti@gmail.com 


\title{
Resumo
}

Esse artigo busca contribuir para a discussão sobre a ontologia e especificidade do trabalho cinematográfico diante de sua transformação tecnológica radical. Para tanto, busco analisar o modo como a tensão entre os mecanismos que promovem a circulação do filme e os parâmetros midiáticos de sua identidade é articulada por meio de uma dupla codificação, simultaneamente algorítmica e jurídica. Essa análise se vale de uma leitura das obras Download finished (Sven König \& !mediengruppe bitnik, 2006) e Artisa sem ideia (Cezar Migliorin, 2005) atenta às suas condições materiais de existência em rede.

Palavras-chave: filme digital; circulação; performatividade midiática; codecs; copyright.

\begin{abstract}
This paper aims to contribute to the ongoing ontological discussion about the specificities of the cinematographic work related to its radical technological transformation. To do so, I analyze how the tension between the mechanisms underpinning the circulation of a movie and the media parameters enforcing its identity is articulated by the means of a double codification, simultaneously algorithmic and legal. This analysis resorts to a close reading of the works Download finished (Sven König \& !mediengruppe bitnik, 2006) and Artisa sem ideia (Cezar Migliorin, 2005), paying attention to the material conditions of their network existence.
\end{abstract}

Keywords: digital movie; circulation; media performativity; codecs; copyright. 
Introdução

A noção de um pós-cinema, conforme aventada popularmente e proposta pelos editores dessa edição especial de REBECA, nos convida a pensar o trabalho cinematográfico para além da sua definição material e mesmo morfológica. O filme sem filme já é a norma. Não se trata de um paradoxo filosófico, mas de uma trivialidade com que a maior parte dos espectadores convive cotidianamente, até mesmo em salas de projeção, mas principalmente fora delas. $O$ trabalho cinematográfico hoje existe encapsulado em arquivos digitais que nada têm a ver com os tradicionais rolos de película. Terá chegado a hora de contemplar a possibilidade de um filme sem imagens - um objeto midiático puramente convencional, sem qualquer marcador específico de forma?

Busco encaminhar essa questão com base em uma análise dos modos pelos quais os filmes são codificados algorítmica e juridicamente. Pretendo demonstrar como essa dupla codificação assegura a coerência objetiva do trabalho cinematográfico ao mesmo tempo em que viabiliza a sua dispersão circulatória. Com isso, levanto a hipótese de que a mera codificação poderia constituir parâmetros de propriedade suficientes para assegurar o pertencimento de um objeto ao meio, desvinculando a especificidade cinematográfica de qualquer outra característica supostamente essencial ou intrínseca.

Meu argumento se vale de um par de obras que recorrem a dispositivos convencionalmente entendidos como gerenciais - codecs e contratos - e os mobilizam como parte operante de suas estratégias expressivas. Nessa inversão, elas nos permitem apreender os negativos da circulação cinematográfica e vislumbrar as conturbadas realidades de individuação tecnológica do meio.

\section{Dados cifrados}

Graças às tecnologias digitais, os filmes parecem ter se emancipado de quaisquer canais e práticas materiais específicas. Assim como outras formas de conteúdo, eles agora circulam como dados que podem ser armazenados, transmitidos e acessados por meio de qualquer equipamento compatível. Contanto que haja um suporte físico para carregar esses dados, o software necessário para executá-los e as interfaces de saída adequadas, o filme poderá aparecer. Essas condições se tornam cada vez mais triviais com o desenvolvimento de uma infraestrutura de computação global. Ao diligenciar o deslocamento de imagens em movimento por diferentes sítios geográficos e institucionais, as redes digitais reduzem operações midiáticas que costumavam ser custosas, inconvenientes e demoradas a tarefas descomplicadas, praticamente instantâneas. Hoje, com um par de cliques do mouse, se pode alugar um longa, preparar um programa de exibição ou enviar a cópia de um filme para uma mostra. 
A superação dessas barreiras propaga a ilusão de imaterialidade. Na aceleração dos fluxos de informação, o filme se desvencilha do meio físico e se mantém coeso no arquivo computacional discretizado. $\mathrm{O}$ cinema percorreu um longo caminho desde os rolos de filme e videotapes. Uma sequência nomeada de bits parece ser tudo de que precisamos atualmente para dar conta de uma instância completa do trabalho cinematográfico.

Mas essas impressões são tão errôneas quanto sedutoras. Existe uma materialidade inegável na computação. Os sistemas subjacentes aos meios digitais não são aparelhos impalpáveis, de constituição metafísica, mas sim um "mundo bagunçado de matéria e metal" (KIRSCHENBAUM, 2008: 27). Friedrich Kittler (1995) nos lembra de que os padrões de bits que constituem informação num computador dão conta das diferenças de voltagem no seu âmago. Devido a esse caráter diferencial da inscrição digital, os dados nunca estão verdadeiramente "armazenados" num disco rígido; eles resultam, em vez disso, de um percurso de energia pelo aparelho (KIRSCHENBAUM, 2008: 95). Na sua dimensão mais concreta, não há nada separando o filme de qualquer outra coisa na máquina. O próprio arquivo digital é uma abstração criada pelo sistema operacional enquanto roda. Toda informação audiovisual que ele supostamente contém é um efeito da atividade sistêmica. $O$ trabalho precisa ser montado por meio de sua interação em tempo real com outras abstrações de software, tais como o aplicativo de reprodução e os drivers de áudio e vídeo. O arquivo não "significa" nada sem um sistema capaz de decodificá-lo. Ele mal existe.

A codificação em vídeo digital simultaneamente intensifica e oculta o caráter performativo do filme. Os algoritmos empregados nessa operação, chamados codecs, organizam sons e imagens perceptíveis de acordo com uma lógica espacial complexa de modo a otimizar sua armazenagem e transmissão (MACKENZIE, 2008: 48). Codecs frequentemente operam por meio de compressão com perdas [lossy compression], que se livra de informações abaixo de um certo limiar de percepção de modo a salvar espaço de memória. DVDs só se tornaram um meio viável para a distribuição de filmes em massa graças à economia propiciada pelo MPEG-2, um codec capaz de reduzir o tamanho de um arquivo de vídeo em até $97 \%$ sem causar qualquer perda notável de qualidade (LASICA, 2005: 88). Com o progressivo aumento da complexidade dos sistemas de computação e do volume de dados em circulação, os codecs se tornaram um componente indispensável da operação de mídias digitais. Ao estabelecer um procedimento padrão para que diferentes máquinas e aplicativos software possam dar conta dos mesmos dados audiovisuais de igual maneira, os codecs viabilizam uma camada comum de atividades midiáticas. Sobre esse chão, os usuários trabalham sem consciência da materialidade da inscrição digital, empregando interfaces que simulam 
as práticas, ferramentas e convenções tradicionais do cinema. Realizadores podem operar como sempre fizeram. Seu trabalho termina onde o dos codecs começa: no empacotar e desempacotar de dados em complexos arranjos de significado.

$\mathrm{Na}$ medida em que depende dessas conveniências operacionais, a circulação de filmes está condicionada pela codificação digital. Kittler (2008) aponta para o fato de que o código computacional está baseado em sistemas criptografados de comando e comunicação que operam por meio da reorganização de informações de acordo com uma convenção particular dentro das convenções universais da linguagem. $O$ acesso à mensagem fica restrito àqueles que dominam a cifra. Em outras palavras, um dispositivo precisa possuir os codecs necessários instalados para poder apresentar o filme da maneira adequada. De outra forma, executar o arquivo de dados resultaria apenas num frame vazio ou numa mensagem de erro. O filme, por sua vez, precisa ser codificado no formato apropriado de modo a ser reconhecido por certos aparelhos. DVDs de vídeo pedem imagens inscritas em H.262/MPEG-2 Part 2 com uma taxa de informação de até 9.8 Mbit/s. O Digital Cinema Package (DCP) especificado pela Digital Cinema Iniciative emprega diferentes containers tipo Material eXchange Format com trilhas de vídeo codificadas em JPEG-2000. Desde o fim de 2016, a Netflix adotou a compressão royaltyfree VP9 (perfil 0), desenvolvida pela Google, para todo conteúdo a ser baixado em dispositivos Android. Esses algoritmos não apenas governam os modos de acesso e aparecimento do trabalho em cada uma de suas plataformas de exibição, mas constituem aspectos formais e operacionais das imagens em movimento. Ao restringir 0 modo como os dados audiovisuais podem ser arbitrariamente organizados para serem reconhecidos como tais, a codificação efetivamente opera como um critério de pertencimento, à guisa de um protocolo.

Alexander Galloway caracteriza um protocolo como "um conjunto de recomendações e regras que delineia padrões técnicos específicos" (GALLOWAY, 2004: 6) existente no cerne da computação em rede. Em princípio, o protocolo é uma tecnologia de inclusão, que permite aos participantes em conformidade que se conectem uns aos outros e formem uma rede que até então não existia. Ao mesmo tempo, o protocolo constitui um importante meio regulatório entranhado no próprio sistema que ele viabiliza. Na medida em que estabelece as condições de existência na e da rede, o protocolo não pode ser confrontado dentro de seus perímetros. Opor-se ao protocolo, adverte o autor, "é como se opor à gravidade" (GALLOWAY, 2004: 147). A codificação de vídeo determina um campo similar de possibilidade nas redes de cinema digital. Ela delineia a forma como os dados devem ser organizados de modo a contar como trabalho cinematográfico para os aplicativos e dispositivos-padrão. Essas restrições amarram a arquitetura fragmentada da computação numa plataforma de comunicação inteligível, 
em continuidade com as práticas midiáticas estabelecidas. A coerência resultante do sistema permite "os usos profundos e significativos que são feitos dele todos os dias" (ibid.) - tais como assistir a um filme. Ao mesmo tempo, ela define os objetos que são próprios para esses usos. O que não está codificado como um filme, ainda que se pareça com um, não será um filme. Ilhas de edição não irão importá-lo, reprodutores de mídia não irão reproduzi-lo, festivais de cinema não irão aceitá-lo como parte de uma inscrição. Ao determinar esses parâmetros, a codificação parece abrir lugar para um predicado de especificidade em nosso mundo pós-midiático. Ela impõe uma identidade ao cinema mesmo quando os filmes já não são em nada diferentes de outros bits circulando pelas redes de computação universal.

Não obstante, a paisagem midiática macia que a codificação produz não é verdadeiramente estável nem duradoura. Ela é continuamente urdida pelos mesmos processos maquínicos que busca velar. Mesmo as mensagens de erro, por mais desconcertantes que possam ser, são efeitos deliberados contribuindo para essas circunstâncias de normalidade. Lá no fundo do computador, não existem filmes "certos" ou "errados", existem apenas as capacidades materiais da inscrição digital. A codificação de interrupções significativas no sistema produz uma aparência de integridade que esconde a arbitrariedade dessa distinção. Isso impede o sistema de rodar um arquivo que ele aceite como um filme, mas que não parecerá um filme ao espectador. Essa dissociação entre a identificação sistêmica dos dados e o fenômeno sensorial que deles resulta desvela a farsa da codificação. A máquina não está intrinsecamente atrelada às expectativas midiáticas dos usuários. A estética ambígua dos artefatos de compressão nos serve como evidência. Um dos "defeitos" mais frequentes na decodificação de imagens em movimento é a perda de quadros-chave de referência (chamados de l-frames), levando o sistema a aplicar a uma cena um padrão de movimento que deveria pertencer a outra (uma sequência de $P$-frames). Massas de pixels se movimentam desconectadas da figura que supostamente representam. 0 computador não parece estar trabalhando corretamente, mas ele está. Em verdade, esses efeitos podem ser produzidos intencionalmente e controlados com relativa precisão durante o processo de codificação. Os artistas associados à cena da glitch art exploram esses processos de compressão de imagem como fonte de efeitos visuais, e muitos realizaram scripts e tutoriais para compartilhar suas técnicas com uma comunidade mais ampla.

Uma iniciativa do tipo é Download finished, projeto criado por Sven König e !mediengruppe bitnik em 2006. Ele consiste num site que convida o público a se apropriar de vídeos "encontrados" na internet como uma modalidade de realização artística. Por meio da plataforma, os usuários podem procurar filmes em redes de troca 
ponto-a-ponto e convertê-los automaticamente em novas obras. O sistema emprega um script que altera a sequência de quadros do filme, ocasionando diversos erros de compressão durante o processo de cópia. $O$ arquivo resultante é em seguida disponibilizado para download. Trata-se de uma sequência de imagens completamente diferente do filme original, e se apresenta de maneira correspondente. Nesse sentido, da mesma maneira que outras práticas de found footage, Download finished interroga as formas sancionadas de intervenção no meio, os modos como se constitui a autoria e as extremidades identitárias do trabalho cinematográfico. Ele recorre a atividades subjacentes ao aparato computacional na mesma medida em que desarticula o arranjo midiático. Ao fazê-lo, ressalta os metaprogramas científicos e industriais frequentemente escondidos sob a circulação de filmes, tais como "[>2] o trabalho dos matemáticos que estabeleceram as condições teóricas para [ $>3$ ] os programadores que criaram o software de codificação / o codec" (KÖNIG; !MEDIENGRUPPE BITNIK, 2007).

Ao aproximar a codificação do vídeo da transmissão de pacotes de dados em redes ponto-a-ponto, Download finished acentua a similaridade entre esses sistemas. Nas imagens reinterpretadas, vislumbramos os modos não-humanos, praticamente alienígenas, pelos quais os codecs performam as imagens em movimento. Trata-se de estruturas de movimento baseadas antes no uso eficiente dos recursos de rede do que na captura óptica linear ou na projeção mecânica. Quanto mais arbitrária é a correspondência entre os dados codificados e a imagem resultante, mais ambientais as operações de codificação precisam ser. Ao mesmo tempo em que libertam o filme de suportes físicos individuais, os meios digitais o entranham ainda mais nos canais de circulação. As condições nítidas de propriedade estabelecidas pela codificação se desdobram no modo como os objetos cinematográficos se dão ao controle. Esse regime de governança tem a sua expressão maior na soberania das plataformas de webstreaming e video-on-demand, capazes de banir usuários específicos, derrubar conteúdo e até mesmo bloquear o acesso a partir de territórios inteiros (LOBATO; MESSE, 2016). As condições algorítmicas de propriedade implicam negociações cada vez mais complexas entre a existência do filme e o acesso aos seus canais de circulação.

Matthew Kirschenbaum afirma que todos os objetos digitais estão fadados a "serem inexoravelmente reduzidos a blocos de código opaco, ou BLOBs, conforme se destacam e derivam para longe de seus ambientes de software nativos" (KIRSCHENBAUM, 2008: 234). Conforme a ecologia de meios se altera, a circulação de filmes digitais só pode continuar graças à manutenção de sistemas capazes de decodificar os dados empregados. Se essa estrutura não estiver disponível nem puder ser emulada de alguma maneira, o filme precisará ser traduzido em um novo formato ou 
virá a se tornar aquilo que, nas palavras de Paolo Cherchi Usai (1999: 46), equivale a um "hieróglifo" indecifrável. Ressaltando a dimensão "fundamentalmente social" dos objetos digitais, Kirschenbaum (2008: 21) vê esse processo de tradução acontecendo antes por meio da apropriação informal do público do que por medidas oficiais das instituições. Nas múltiplas transcodificações realizadas diariamente pelos usuários conforme eles ripam discos ópticos, baixam vídeos do YouTube, gravam imagens da tela de cinema, mudam seu formato e resolução, inscrevem legendas na imagem, copiam arquivos em dispositivos avulsos, extraem cenas para compartilhar nas redes sociais etc. -, podemos perceber uma forma errática de preservação colaborativa. Nesse sentido, as economias da "imagem pobre" descritas por Hito Steyerl (2009) não seriam uma mera expressão de degeneração das imagens em movimento. Pelo contrário, seriam a forma mais frequente (e muitas vezes a única) por meio da qual o filme subsiste.

No horizonte derradeiro dessas transcodificações, surgem instâncias em que a individualidade do filme parece ceder completamente. O corte final, que o estúdio havia editado para exibição doméstica e depois empacotado numa versão definitiva de colecionador, agora se dissolve em um milhão de variantes suplementares, feitas a toda hora, por praticamente qualquer um. Elizabeth Hills (2002) ressalta a multiplicação de fan edits ocasionada pelas redes de computação no começo dos anos 2000 . O acesso a pacotes software similares àqueles usados por estúdios profissionais e a possibilidade de constituir comunidades globais online levaram a interferência do público muito além da videofilia dos anos 1980. Os usuários sujeitos aos protocolos de rede se vêem livres para operar dentro de todas as possibilidades criadas por dados. Como observa Lev Manovich, não são apenas conteúdos midiáticos que podem ser combinados e rearranjados, mas até mesmo "as técnicas fundamentais, os métodos de trabalho e as formas de representação e expressão" de diferentes mídias (MANOVICH, 2013: 268). O público se apropria dos materiais fílmicos e, valendo-se da remixabilidade profunda viabilizada por ambientes software, produz novas versões a partir deles.

Contradizendo a dissociação identitária proposta por Download finished, convido os leitores a apreciarem o quanto de um trabalho cinematográfico persiste em cada uma de suas hibridizações. Vídeos de reação, excertos virais, paródias, remakes e remixes ainda expressam, de certa maneira, o filme "original". Na mesma medida em que eles reconfiguram o trabalho em novas estruturas de sentido, eles proporcionam meios para sua circulação. Não é de surpreender que estas sejam as formas predominantes assumidas por um filme emaranhado nas tramas sociais das redes de computador. Do ponto de vista intermediário proporcionado pela transmissão, o filme existe menos como um objeto midiático individual do que como um contínuo de fluxos 
de informação. Conforme as distinções entre as operações de produção e consumo entram em colapso, mais se parece simultaneamente retirar de e agregar ao filme em cada um de seus aparecimentos parciais. O filme é imbuído de traços de afeto da audiência, destilado em seus elementos mínimos, despido de seus vieses, reinterpretado e rearranjado. Por meio dessas mediações pouco ortodoxas, a identidade auto-diferencial do filme é executada ao mesmo tempo em que se transforma. Sob essas circunstâncias, o trabalho cinematográfico se parece menos com uma totalidade que está sempre fora do nosso alcance do que com um produto de acreções conflitantes uma presença porosa ao circuito, sempre vazando para o ambiente, sempre aberta a novas afluências.

\section{Termos de uso para o consumidor final}

Ação e dispersão (2002) é um documentário em primeira pessoa de Cezar Migliorin. Foi feito sem roteiro, baseado num conjunto de instruções que coincide com sua sinopse: "Um homem e uma câmera. Jamais duas noites na mesma cidade. Até o dinheiro acabar." O dinheiro em questão foram os 20 mil reais recebidos de um edital de apoio da Petrobrás, a petroleira estatal que era uma das principais patrocinadoras do cinema brasileiro na época da realização. Depois de separar dinheiro para pagar imposto, equipamentos e taxas, Migliorin embarcou numa jornada aleatória com o propósito de gastar todo o recurso restante. Os cinco minutos do curta documentam suas viagens do Rio de Janeiro a Penedo e a outras treze cidades por todo o mundo. A cada take, o diretor tensiona os tabus relacionados ao financiamento público para as artes. Vislumbramos quartos de hotel, mesas de restaurante e diversos meios de transporte, enquanto um marcador no canto inferior esquerdo da tela faz a contagem regressiva do dinheiro restante. Cada cena simultaneamente exalta e denuncia o financiamento estatal, numa lembrança constante de como a realização cinematográfica está circunscrita a esse aparelho. Nesse sentido, Migliorin desenvolve uma modalidade de cinema estrutural que se engaja com o meio para além dos dispositivos imediatos do filme e do projetor. $O$ cineasta parece tentar converter o puro valor do edital em imagens de puro sentido, mas acaba produzindo imagens que significam apenas a si mesmas. A crueza de certas cenas esfrega a gratuidade do processo na cara dos espectadores, borrando as linhas entre o gesto criador e a exploração do globetrotting como moeda. Elas parecem propor que o mero gasto de energia na forma de dinheiro, comida e combustível, ao colocar as coisas em movimento, seria o bastante para configurar um filme. 
Em 2004, Ação e dispersão foi contemplado com um prêmio de 4 mil dólares no VIPER Basel International Festival for Film, Video and New Media, na Suíça. Decidido a retornar ao festival no ano seguinte com uma obra competitiva, Migliorin separou metade desse dinheiro para um novo projeto chamado Artista sem ideia (2005). Ele lançou uma convocatória aberta para aquele que seria o seu próximo trabalho. $O$ anúncio convidava outros realizadores a enviarem para ele filmes completos e inéditos de qualquer duração e formato. Cumprido o prazo, Migliorin iria escolher uma das obras para ser sua e pagar ao autor mil dólares em troca da autoria. Os outros mil seriam gastos na promoção e publicidade do filme. De acordo com a convocatória, o trabalho permaneceria completamente inalterado, tendo apenas a linha "um filme/vídeo de Cezar Migliorin" adicionada aos seus créditos finais (MIGLIORIN, 2005b). O filme escolhido e a transação decorrente ficariam em segredo. A intenção declarada de Migliorin era colocar em questão o modo como esse tipo de chamada de trabalho conduz a produção artística. Com sua proposta ambivalente, ele convidava uma audiência de realizadores a refletir sobre os efeitos estéticos que as demandas do circuito podem ter sobre seus trabalhos. Ele os conclamava a resistir ao "fetiche dos créditos" que transforma uma obra de arte em mero veículo para o prestígio do autor. Mas, ao mesmo tempo, ele buscava explorar o cisma entre trabalho e autoria para obter imagens que não fossem "contaminadas pelas ação que as produziu" (MIGLIORIN, 2005b). Uma subjetividade curatorial, supostamente mais pura, atuaria no lugar da do realizador. O enquadramento contratual da convocatória seria a ferramenta usada para apreender o filme.

O recurso de Artista sem ideia aos mecanismos jurídicos subjacentes ao meio não apenas representa um avanço no projeto de cinema estrutural cínico de Migliorin. Ele também expressa uma tendência mais geral. A crescente volatilidade ocasionada pelos sistemas digitais afiou os aparatos legais que controlam a mídia. Como explica Lawrence Lessig (2008), o software computacional é particularmente sujeito às leis de direito autoral. Em virtude da sua arquitetura, a mídia digital gera cópias de cada porção do código durante a sua transmissão ou acionamento. Mesmo o ato de carregar um arquivo numa máquina local envolve a duplicação de dados entre seus componentes internos, da armazenagem para a memória. Cada engajamento com um filme digital dispara, portanto, leis de direito de autor, e "precisa ser justificado por uma licença ou sob a lei do "fair use'" (LESSIG, 2008: 99). Essa justaposição de dispositivos tecnológicos e jurídicos proporciona meios para a governança da circulação de filmes. As operações midiáticas viabilizadas pelo protocolo são constritas, em toda sua extensão, pelo Estado de Direito. Não importa o quanto um filme se disperse na rede: ele permanecerá comprometido por licenças, contratos e termos de uso para o consumidor final. As instruções expedidas pelo detentor do copyright efetivamente 
funcionam como restrições de uso, ocasionalmente implementadas na forma de sistemas de regulação digital de direitos [digital rights management-DRM].

Por esse motivo, os direitos de propriedade intelectual parecem triunfar sobre a própria imagem como condição para o aparecimento do filme. Eles prometem conter não apenas a forma em que o trabalho cinematográfico é disponibilizado no presente, como também todas as suas iterações possíveis e futuras. Algumas instâncias do filme são autorizadas a existir apenas na medida em que se submetem à autoridades do copyright. Mecanismos de autorização legitimam o controle e propriedade de um autor ausente sobre as variações do trabalho realizadas por outrem. Em igual medida, elas asseguram que essas variações serão mobilizadas de modo a reforçar a primazia de originalidade em vez de enfraquecê-la.

Podemos ver esses mecanismos atuando na forma como os estúdios incorporam certas práticas audiovisuais possibilitadas pelas novas mídias. O concurso Official Star Wars fan film awards, realizado pela própria Lucasfilm, oferece espaço para que os filmes realizados por fãs sejam exibidos e celebrados, mas apenas na medida em que eles se submetam a uma série de parâmetros de conteúdo. A participação está condicionada à cessão total de direitos à Lucasfilm em todos os canais de mídia (CHANNEL STAR WARS, 2011). Dessa forma, o estúdio simultaneamente preserva a integridade narrativa da sua propriedade intelectual e explora o valor gerado pelo trabalho da audiência. Seguindo uma lógica similar, companhias contratam artistas como o duo de VJs Addictive TV para realizar remixes de lançamentos blockbuster. Lançadas na internet e exibidas em festas, essas obras têm o objetivo de divulgar a exibição e distribuição do filme para além de seus espaços regulares. Dessa forma, em vez de promover uma desconstrução potencial do trabalho cinematográfico, o remix opera como uma ferramenta para a sua promoção.

Instrumentos institucionais como esses servem para domar interações agonísticas sob um status quo midiático. Eles sancionam certas operações das novas mídias na medida em que as subsumem à rationale estabelecida do cinema. Ao fazê-lo, também reiteram a diferença entre componentes e iterações distintas do trabalho cinematográfico como uma hierarquia ontológica. Ao emular os mecanismos dessa economia de presenças, Artista sem ideia a vira do avesso. A transparência do projeto de Migliorin estimula, em vez de conter, a multiplicidade do filme. Sua convocatória supostamente recebeu mais de cem propostas distintas. Ele disse que foi o filme mais trabalhoso que já realizou. O trabalho de responder a cada uma das mensagens frequentemente o fez sentir-se como "uma empresa, uma organização" (MIGLIORIN, 2005b). Muitas (se não todas) essas interações foram publicadas em um blog, usando um dos templates padrão do Blogspot: sem imagens, texto branco sobre fundo preto. $O$ 
último post é uma reprise - ou um flashback? - da proposta inicial de Artista sem ideia, na forma como ela foi enviada para cerca de 250 pessoas ligadas ao cinema e à arte (MIGLIORIN, 2005a). O imediatamente anterior comunica os resultados do concurso: 0 vídeo escolhido como vencedor foi o de número 111, e o contrato de transferência de direitos foi assinado na presença de duas testemunhas em 20 de abril de 2005.

É difícil encontrar qualquer outro resultado concreto do projeto além dessa breve nota. Não há como saber com certeza qual filme seria esse, entre vários outros de autoria indiscutível na filmografia do diretor. Talvez nenhum. Talvez a convocatória tenha sido uma farsa desde o princípio, números tenham sido manipulados. Quem pode dizer? O caso é que Migliorin saiu em busca de imagens que não tivessem sido contaminadas e parece ter voltado de mãos abanando. Por outro lado, ele disparou uma série de novas contaminações: camadas de diálogo e controvérsias, colunas em suplementos culturais, perguntas, provocações, teorias, análises como esta. Pode ser que, talvez, precisamente nesses rastros sem um corpo - paratextos de um texto inexistente -, o diretor tenha encontrado o trabalho ao qual aspirava: "um filme onde você tivesse dúvidas se se trata de um filme, tal a força com que ele aponta para fora" (ibid.). Trata-se de um objeto que existe não como fenômeno de percepção direta, mas de imaginação pública. Estando em negativo, faz positivas as estruturas subjacentes ao circuito. Circulação contínua sem encerramento.

\section{Considerações finais}

A ideologia midiática nos leva a crer que o trabalho cinematográfico possui uma identidade objetiva, estável e homogênea. Sondar os seus meios de circulação, entretanto, nos revela o contrário. O filme leva uma existência precária, que depende da articulação composta de diversos componentes materiais, tanto fixos quanto móveis. Esse processo é contingente a instruções de operação, relatos discursivos e cláusulas de uso e cópia, tanto dentro quanto fora das práticas midiáticas reconhecidas. O filme está, portanto, sempre em realização. Não é possível circunscrever sua produção apenas às atividades convencionalmente reconhecidas como tal. Não se trata de uma arte de um ou dois estágios, mas de múltiplos. Rearranjos não são apenas esperados, mas a própria condição de existir no circuito. A intensificação da circulação dissolve o filme nas trocas informativas subjacentes. Ao mesmo tempo, carrega forças midiáticas que buscam assegurar que toda ocasião do trabalho represente o mesmo objeto desde sempre ausente. Apelando a parâmetros metafísicos, essas forças ratificam o controle autoral. Ao estabelecer formas, instâncias e sítios privilegiados para o contato com o trabalho, elas desenham a topografia de aparatos ao redor do público. O filme se encontra nesse jogo contínuo entre dispersão e contenção. 


\section{Referências}

CHANNEL STAR WARS. Star Wars fan movie challenge - Guidelines. Atom Comedy

Central Originals. $2011 . \quad$ Disponível em:
http://atom.com/spotlights/starwars/challenge/production_kit.html. Acesso em: 14 ago. 2011.

GALLOWAY, Alexander. Protocol: how control exists after decentralization. Cambridge, MA: MIT, 2004.

HILLS, Elizabeth. 'Use the camcorder Luke': Star Wars fan films and digital moviemaking. In: IT for Regional Culture, Media and The Arts Conference, ago. 2002. Anais [...]. p. 176-182.

KIRSCHENBAUM, Matthew. Mechanisms: new media and forensic imagination. Cambridge, MA: MIT, 2008.

KITTLER, Friedrich. Code. In: FULLER, Matthew. Software studies: a lexicon. Cambridge, MA: MIT, 2008. p. 40-47.

KITTLER, Friedrich. There is no software. CTheory, 18 out. 1995. Disponível em: http://ctheory.net/articles.aspx?id=74. Acesso em: 25 ago. 2018.

KÖNIG, Sven; !MEDDIENGRUPPE BITNIK. what is the characteristic structure of p2p films? Download finished - FAQ, 2007. Disponível em: http://www.downloadfinished.com/faq.html. Acesso em: 25 ago. 2018.

LASICA, Joseph Daniel. Darknet: Hollywood's war against the digital generation. Hoboken, NJ: John Wiley \& Sons, 2005.

LESSIG, Lawrence. Remix: making art and commerce thrive in the hybrid economy. New York City: Penguin Press, 2008.

LOBATO, Ramon; MESSE, James (eds.). Geoblocking and global video culture. Amsterdam: Institute of Network Cultures, 2016.

MACKENZIE, Adrian. Codecs. In: FULLER, Matthew. Software studies: a lexicon. Cambridge, MA: MIT, 2008. p. 48-54.

MANOVICH, Lev. Software Takes Command. London: Bloomsbury, 2013.

MIGLIORIN, Cezar. O resultado. Artista sem ideia, 11 abr. 2005a. Disponível em: http://artistasemideia.blogspot.com/2005/04/o-resultado.html. Acesso em: 25 ago. 2018.

MIGLIORIN, Cezar. Projeto Artista sem ideia. Canal Contemporâneo, 27 jan. 2005b. Disponível em: http://canalcontemporaneo.art.br/saloesepremios/archives/000356.html. Acesso em: 25 ago. 2018.

STEYERL, Hito. In defense of the poor image. e-flux, 10 nov. 2009. Disponível em: http://e-flux.com/journal/10/61362/in-defense-of-the-poor-image. Acesso em: 25 ago. 2018. 
USAI, Paolo Cherchi. Decay cinema: the art and aesthetics of moving image destruction. Stanford Humanities Review, vol. 7.2, jul. 1999. p. xiv-49. 